\title{
ANÁlISE DA RESPIRAÇÃO MITOCONDRIAL EM TECIDO CEREBRAL DE GATO APÓS ISQUEMIA E REPERFUSÃO ${ }^{1}$
}

\author{
ANALYSIS OF MITOCHONDRIAL RESPIRATION IN BRAIN \\ CEREBRAL TISSUE OF CATS AFTER ISCHEMIA AND \\ REPERFUSION
}

\author{
Hiroshi Nakano ${ }^{2}$ \\ Benedicto Oscar Colli ${ }^{3}$ \\ José Eduardo de Salles Roselino ${ }^{4}$
}

\section{RESUMO}

Introdução - A isquemia cerebral é uma doença freqüente e de difícil tratamento médico. De particular interesse neurocirúrgico são as situações de vasoespasmo após hemorragia subaracnóidea, de oclusão temporária de vasos nas neurocirurgias e de tromboses de artérias intracranianas. A lesão cerebral resultante da isquemia depende da sua duração e pode ser agravada pela reperfusão do território isquêmico. Vários estudos clínicos e experimentais têm sido realizados para melhor entender esses fenômenos. Objetivo - Este trabalho visou a avaliação precoce dos efeitos da isquemia focal seguida da reperfusão no cérebro de gatos. Métodos - A isquemia cerebral foi provocada por clipagem temporária da artéria cerebral média por tempos determinados com reperfusão durante 10 minutos, e avaliação foi efetuada através da análise da respiração mitocondrial no tecido isquemiado. Resultados - Houve redução significativa no consumo de $\mathrm{O} 2$ nas amostras de tecido cerebral isquemiado por 60 minutos, seguidos de 10 minutos de reperfusão, quando comparadas ao tecido cerebral contralateral (não isquemiado). Conclusões - Com base nos resultados obtidos, pode-se concluir que o tempo de duração da isquemia foi um fator determinante na alteração da respiração mitocondrial de gatos submetidos à isquemia e reperfusão de curta duração (alterações significativas apenas após 60 minutos de isquemia seguidos de 10 de reperfusão). Disponível em URL: http://www.scielo.br/acb

Descritores - isquemia, reperfusão, respiração mitocondrial.

\begin{abstract}
Objective - Brain ischemia is considered a disease difficult to be treated. Despite many other clinical situations, of particular interest for neurosurgery is its occurrence in cerebral vasoespam following subarachnoid hemorrhage, in temporary occlusion of intracranial vessels during neurosurgeries and, in intracranial arterial thrombosis. The cerebral lesion caused by isquemia is time-related and it can aggravated by the reperfusion of the ischemic site. Many clinical and experimental studies have been perfomed aiming the better understanding of these phenomena. This study aimed to analyse the precocious effects of focal isquemia and reperfusion uppon the brain of cats. Methods - Focal brain ischemia was performed by temporary clipping of the middle cerebral artery for determined times followed by reperfusion during 10
\end{abstract}

1 Trabalho realizado no Laboratório de Técnica Cirúrgica e Cirurgia Experimental do Departamento de Cirurgia e Anatomia da Faculdade de Medicina de Ribeirão Preto - Universidade de São Paulo como parte dos requisitos para obtenção do Título de Mestre.

2 Neurocirurgião, Assistente de Ensino da Faculdade de Medicina de Marília, Pós-Graduando da Área de Clínica Cirúrgica Faculdade de Medicina de Ribeirão Preto - USP.

3 Professor Titular do Departamento de Cirurgia e Anatomia, Chefe da Disciplina de Neurocirurgia da Faculdade de Medicina de Ribeirão Preto - Universidade de São Paulo.

4 Professor Associado do Departamento de Bioquímica e Imunologia da Faculdade de Medicina de Ribeirão Preto - Universidade de São Paulo. 
minutes. The effects of isquemia were assessed through mitochondrial respiration analysis in the ischemic tissue. Results - The results showed a significant decrease in $\mathrm{O}_{2}$ consumption in samples of brain tissue submitted to 60 minutes of ischemia and 10 minutes of reperfusion when compared with not ischemic brain tissue, indicating compromising of the mitocondrial function. Conclusion - Based on the results we can conclude that time of ischemia was a determinant factor in the mitochondrial respiration alterations in brain tissue of cats submitted to ischemia and reperfusion of short duration (significant alterations observed only after 60 minutes of ischemia followed by 10 minutes of reperfusion).

Key Words - ischemia, reperfusion, mitocondrial respiration.

\section{INTRODUÇÃO}

O acidente vascular cerebral é uma doença de grande relevância médica. Em 1998 nos Estados Unidos da América ocorreram 600.000 casos novos ou recorrentes com custo direto e indireto estimado em 43,3 bilhões de dólares ${ }^{1}$. O acidente vascular cerebral isquêmico (A.V.C.I.), pode ocorrer tanto em doenças primárias, como secundariamente a doenças sistêmicas, e, devido à sua etiologia multivariada, é uma doença de difícil tratamento. O A.V.C.I. pode ser global ou focal. Um exemplo típico de isquemia focal é a oclusão de um determinado vaso; na isquemia global todo cérebro é acometido como, por exemplo, nas assistolias e na hipotensão arterial prolongada ${ }^{2}$. O neurocirurgião está particularmente habituado com algumas situações em que ocorrem isquemias focais, como o vasoespasmo na hemorragia subaracnóidea, a clipagem temporária de artérias em cirurgias vasculares cerebrais.

A conseqüência do AVCI é a lesão cerebral decorrente da isquemia sofrida no território atingido. A gravidade da lesão cerebral durante a isquemia depende do seu tempo de duração, da localização da oclusão vascular, da presença de circulação colateral e de medidas de proteção ao cérebro isquêmico. Outros fatores que contribuem para a gravidade da lesão são a hiperglicemia, a elevação da temperatura e a acidose. Além disso, acredita-se que a reperfusão do tecido isquemiado contribua para agravar os efeitos da lesão isquêmica.

Estudos clínicos e experimentais tem sido realizados para entender melhor a fisiopatologia dos processos de isquemia e reperfusão. Os estudos clínicos e epidemiológicos da isquêmia cerebral em humanos, geralmente são multicêntricos e as vezes suas conclusões não são muito bem definidas, tornando-os limitados a poucos tópicos de estudo ${ }^{3}$. Os estudos experimentais apresentam como vantagens, a possibilidade de um melhor controle das variações biológicas, a possibilidade de utilização de métodos mais invasivos e a mensuração mais rigorosa dos resultados. Como desvantagem apresentam a dificuldade de extrapolar os resultados obtidos em animais de laboratório para os seres humanos.

O objetivo deste estudo foi avaliar as alterações da isquemia cerebral seguida de reperfusão, através da respiração mitocondrial, em um modelo experimental em gatos.

\section{MÉTODOS}

Os experimentos cirúrgicos e a análise da respiração mitocondrial foram realizados no Laboratório de Técnica Cirúrgica e Cirurgia Experimental do Departamento de Cirurgia e Anatomia da Faculdade de Medicina de Ribeirão Preto da Universidade de São Paulo.

Foram utilizados 40 gatos adultos, pesando entre 32 e $3 \mathrm{Kg}$, sem raça definida. Os animais foram divididos em 4 grupos de 10 (Grupos I, II, III e IV).

Os gatos foram anestesiados com thionembutal $30 \mathrm{mg} / \mathrm{kg}$ E.V., (respiração assistida com entubação endotraqueal e respiração mecânica com respirador Takaoka, com parâmetros ajustados para manter a $\mathrm{pCO}_{2}$ entre 34 e $42 \mathrm{mmHg}$ ). Durante o procedimento foram colhidas amostras de sangue para exames de gasometria e glicemia. A temperatura foi mantida entre 37 e $38^{\circ} \mathrm{C}$, entretanto, problemas técnicos com o termistor utilizado, constatados no final do experimento, não permitiram que a medida da temperatura pudesse ser considerável confiável em todos os animais. A pressão arterial média foi mantida entre 80 e $90 \mathrm{mmHg}$, efetuando-se injeções de pequenas doses de thionembutal quando o animal começava a apresentar sinais de superficialização da anestesia.

$\mathrm{O}$ acesso à artéria cerebral média direita foi efetuado através de craniectomia intradural retro-orbital ampliada (técnica de Van Der Sprenkel \& Tulleken ${ }^{3}$ modificada). Após a craniectomia os procedimentos foram realizados sob o microscópio cirúrgico. A duramáter foi aberta em toda a extensão da craniectomia e rebatida sobre o teto da órbita. O lobo frontal foi discretamente deslocado em sentido súpero-medial e a artéria carótida interna foi identificada sobre o nervo óptico e isolada. Em seguida a artéria cerebral média foi identificada e clipada logo após a sua emergência da artéria carótida interna, com um clipe de aneurisma (clipe para clipagem temporária Yasargil FD 710, força 110gms, Aesculap-Germany) (Figura 1). 


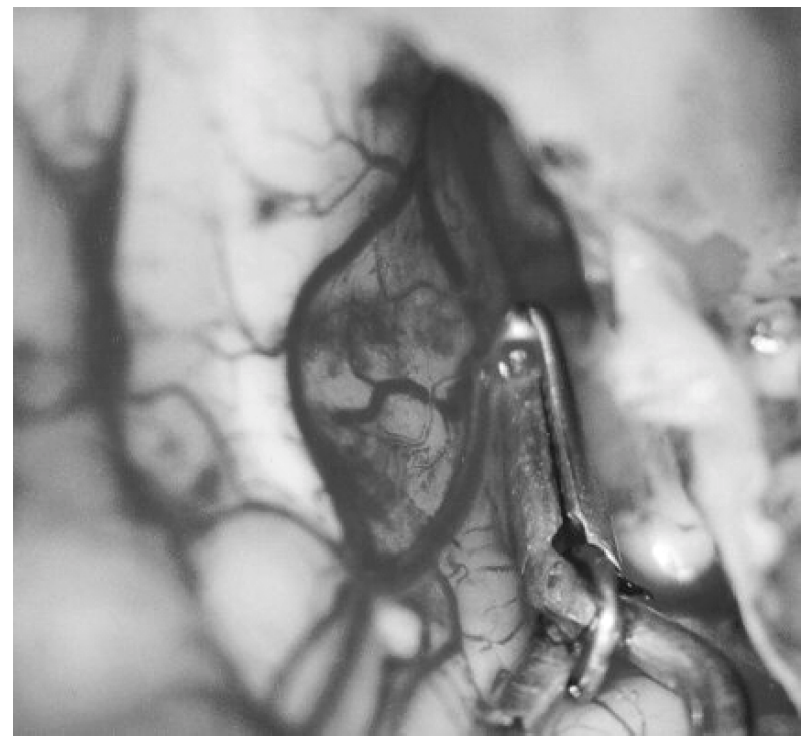

Figura 1 - Exposição intra-operatória da artéria cerebral média direita do gato após aplicação de um clipe de aneurisma (seta).

Os animais dos grupos I, II, e III foram submetidos respectivamente à isquemia por 15,30 e 60 minutos e posteriormente reperfundidos por 10 minutos. $\mathrm{O}$ grupo IV (grupo "sham"), foi submetido somente à craniectomia e à abertura da dura-máter.

\section{Análise da Respiração Mitocondrial}

Após o procedimento cirúrgico foram colhidas amostras de tecido cerebral do opérculo frontal direito (isquemiado) e de tecido cerebral do opérculo esquerdo (não isquemiado) para análise da respiração mitocondrial.

As amostras de tecido cerebral foram lavadas com soro fisiológico por $2 \mathrm{x}$ a uma temperatura de $0 \mathrm{a} 4^{\circ} \mathrm{C}$, e as mitocôndrias foram isoladas através de centrifugação diferencial ${ }^{4,5}$.

$\mathrm{O}$ consumo de $\mathrm{O}_{2}$ pelas mitocôndrias em relação aos substratos piruvato/malato foi determinado polarograficamente utilizando-se o Oxígrafo de Gilson (Medical Eletronics - USA), acoplado a um eletrodo de Clarck. Foram determinadas as velocidades de consumo de oxigênio dos estado III e IV da respiração mitocondrial e a RCR. O estado IV foi obtido adicionando-se $2 \mathrm{mg} / \mathrm{ml}$ de proteína mitocondriais a $1,3 \mathrm{ml}$ do meio de respiração e o estado III adicionando-se 200 moles de MgADP ao estado IV. Para obtenção dos valores dos Estados III e IV da respiração mitocondrial, duas retas foram traçadas sobre as curvas destes estados e duas outras retas paralelas aos eixos $\mathrm{X}$ e $\mathrm{Y}$ (Figura 2) foram traçadas para, juntamente coma as anteriores, formarem um triângulo retângulo. $\mathrm{O}$ cateto deste triângulo paralelo ao eixo das abscissas deve medir $1,5 \mathrm{~cm}$, que correspondia a 1 minuto do traçado. A extensão do outro cateto pode ser transformada em valores expressos em nanomoles de $\mathrm{O}_{2}$ consumidos por minuto. Como a quantidade de mitocôndrias colocada na câmara de $\mathrm{O}_{2}$ foi de 2,0mg, dividindo-se o valor encontrado anteriormente por 2 , a respiração mitocondrial passou a ser expressa em nanoátomos de $\mathrm{O}_{2} /$ minuto/mg de mitocôndria.

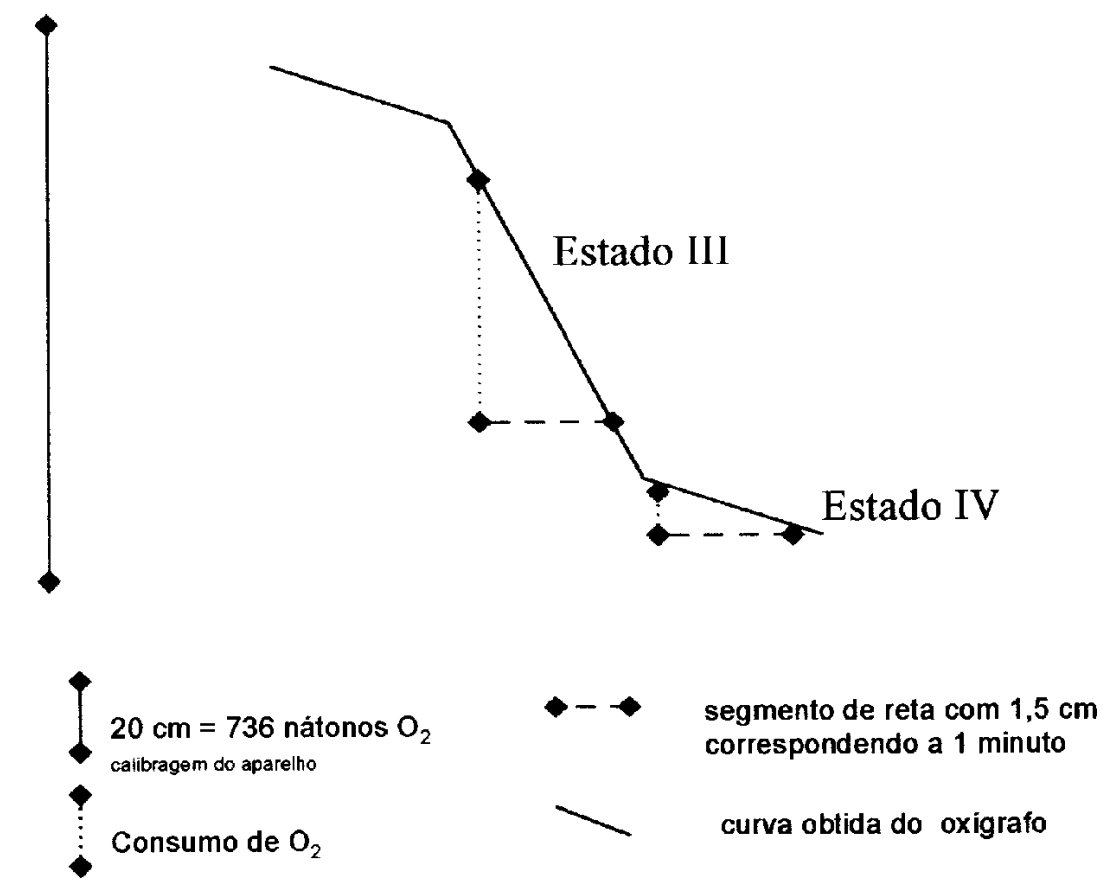

Figura 2 - Curva de Respiração Mitocondrial 


\section{Análise Estatística}

Os resultados dos parâmetros da respiração mitocondrial (Estado III, Estado IV e RCR), foram comparados, utilizando-se o teste não paramétrico de Wilcoxon para amostras pareadas, adotando-se um nível de significância $p<0,05$ quando comparadas as duas amostras do mesmo animal (hemisférios direito e esquerdo). Para a comparação das áreas isquêmicas nos diferentes grupos (áreas corticais direitas), foi utilizado o teste não paramétrico de Kruskal-Wallis, ao nível de significância $\mathrm{p}<0,05$.

\section{RESULTADOS}

Os resultados obtidos em relação aos parâmetros da respiração mitocondrial (Estado III, Estado IV e RCR) são apresentados na tabela 1 .

Tabela 1 - Média e desvio padrão do consumo de $\mathrm{O}_{2}$ pelas mitocôndrias em nmoles $/ \mathrm{min} / \mathrm{mg}$ nos estados III e IV da respiração mitocondrial e razão de controle respiratório (RCR) mitocondrial nos animais dos 4 grupos experimentais.

\begin{tabular}{|c|c|c|c|c|}
\hline & & $\begin{array}{c}\text { Respiração } \\
\text { Estado III } \\
\end{array}$ & $\begin{array}{c}\text { Respiração } \\
\text { Estado IV }\end{array}$ & RCR \\
\hline \multirow[t]{6}{*}{ Grupo 1} & Médias & & & \\
\hline & $\mathrm{Ce} 1$ & 48,60 & 13,84 & 3,51 \\
\hline & $\mathrm{Ce} 2$ & 56,07 & 14,18 & 4,70 \\
\hline & Desvio Padrão & & & \\
\hline & $\mathrm{Ce} 1$ & 48,08 & 13,06 & 0,81 \\
\hline & $\mathrm{Ce} 2$ & 43,24 & 13,42 & 2.10 \\
\hline \multirow[t]{6}{*}{ Grupo 2} & Médias & & & \\
\hline & $\mathrm{Ce} 1$ & 61,82 & 18,58 & 3,79 \\
\hline & $\mathrm{Ce} 2$ & 67,62 & 18,32 & 4,11 \\
\hline & Desvio Padrão & & & \\
\hline & $\mathrm{Ce} 1$ & 24,16 & 9,10 & 1,64 \\
\hline & $\mathrm{Ce} 2$ & 22,49 & 9,34 & 1,12 \\
\hline \multirow[t]{6}{*}{ Grupo 3} & Médias & & & \\
\hline & $\mathrm{Ce} 1$ & 55,43 & 12,72 & 5,41 \\
\hline & $\mathrm{Ce} 2$ & 69,98 & 10,88 & 7,09 \\
\hline & Desvio Padrão & & & \\
\hline & $\mathrm{Ce} 1$ & 20,54 & 6,07 & 3,20 \\
\hline & $\mathrm{Ce} 2$ & 19,05 & 3,80 & 3,00 \\
\hline \multirow[t]{6}{*}{ Grupo 4} & Médias & & & \\
\hline & Direito & 51,08 & 11,86 & 4,23 \\
\hline & Esquerdo & 47,31 & 13,04 & 3,83 \\
\hline & Desvio Padrão & & & \\
\hline & Direito & 36,21 & 2,55 & 2,35 \\
\hline & Esquerdo & 21,47 & 3,48 & 1,89 \\
\hline
\end{tabular}

Obs: $\quad$ Ce $1=$ Amostra do Hemisfério Direito Isquêmico)

Ce 2 = Amostra do Hemisfério Esquerdo (Controle)

Houve uma diferença significante no consumo de $\mathrm{O}_{2}$ no estado III nas amostras do tecido cerebral dos animais submetidos a 60 minutos de isquemia e $10 \mathrm{~m}$ de reperfusão (grupo III) quando comparadas ao tecido cerebral contralateral não isquemiado $(\mathrm{p}=0,02)(\mathrm{Fi}-$ gura 3). Não houve diferença significante entre os consumos de $\mathrm{O}_{2}$ nos estados III nas amostras isquêmicas e não isquêmicas nos animais dos grupos I,II e IV. 


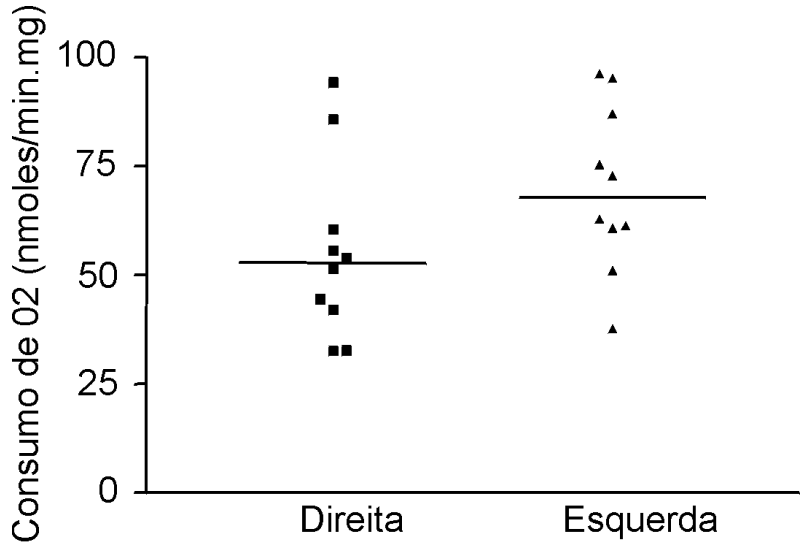

Amostras de Tecido Cerebral

Figura 3 - Consumo de $\mathrm{O}_{2}$ pelas mitocôndrias nas áreas isquêmicas (direita) e não isquêmica (esquerda) no estado III nos animais do grupo III (60 minutos de isquemia e 10 minutos de reperfusão). Barras horizontais $=$ medianas.

Não houve diferença significante entre os consumos de $\mathrm{O}_{2}$ no estado IV e entre as RCR nas amostras isquêmicas e não isquêmicas nos animais dos grupos I a IV.

A comparação dos consumos de $\mathrm{O}_{2}$ nos estados III e IV e entre as RCR nas amostras de tecido do hemisfério cerebral direito do grupo IV ("sham") e nas áreas isquêmicas (direita) dos animais dos grupos I a III não mostrou diferença significante entre nenhum destes parâmetros.

\section{DISCUSSÃO}

A dificuldade de se conseguir uma terapia eficaz para a isquêmia cerebral e os problemas éticos, técnicos e ao alto custo para se fazer observações dos fenômenos isquêmicos em seres humanos, tem induzido os pesquisadores a procurar conhecer melhor essas alterações em experimentos laboratoriais.

No presente experimento o gato foi utilizado como animal de experimentação pela seguintes razões: facilidade para anestesiar e manter a respiração controlada, facilidade para os controles necessários (gasimetria, pressão arterial média, glicemia, temperatura) e por ser um animal de porte médio, cuja artéria cerebral média, mostrou-se adequada para os procedimentos cirúrgicos de isolamento e clipagem temporária para produzir a isquemia e reperfusão. Como desvantagem, por não ser um animal de cativeiro, houve dificuldade de determinação da idade dos animais e de separar os animais mais velhos dos mais jovens, que provavelmente apresentam diferentes condições da árvore circulatória.

A manutenção das condições de homeostase do animal é essencial para a verificação das alterações provocadas por isquemias focais transitórias ${ }^{6,7}$. Os principais fatores que interferem na gravidade do fenômeno são a temperatura elevada, a acidose e a glicemia elevada ${ }^{7}$. O controle dos gases sangüíneos em nosso estudo pode ser efetuado de maneira adequada com a metodologia empregada para a realização da anestesia dos animais e as glicemias observadas no início do procedimento foram normais. Por problemas técnicos com o sensor para detecção da temperatura constatados na fase final do experimento, o controle deste parâmetro não pode ser considerado completamente confiável em todos os animais.

Por ser a mitocôndria a principal responsável pela produção de energia, a avaliação da sua função pode ser importante no estudo das alterações celulares precoces causadas pela isquemia cerebral. A preservação da função após um fenômeno isquêmico poderá ser um bom indicativo de que o sofrimento cerebral não teria sido importante.

Vários estudos têm sido efetuados no Laboratório de Técnica Cirúrgica e Cirurgia Experimental do Departamento de Cirurgia e Anatomia da Faculdade de Medicina de Ribeirão Preto da Universidade de São Paulo empregando-se o estudo da função mitocondrial para avaliar os efeitos da isquemia tecidual ${ }^{8-11}$, inclusive sobre o tecido cerebral do rato ${ }^{12,13}$. Recentemente esta metodologia foi empregada em nosso laboratório para avaliar o efeito da isquemia no tecido cerebral de gatos submetidos à isquemia focal transitória obtida por oclusão da artéria cerebral média efetuada por abordagem direta do vaso ${ }^{14}$. Os resultados obtidos mostraram que não houve alteração significativa nos parâmetros da respiração mitocondrial nos diferentes grupos de animais submetidos a períodos de até 60 minutos de isquemia. A ausência de alterações na função mitocondrial foi atribuída a um possível efeito neuroprotetor do anestésico utilizado ${ }^{11,15}$, no caso a quetamina

No presente estudo, delineado de maneira semelhante ao anterior, acrescentando-se a reperfusão da área isquêmica durante 10 minutos, os resultados mostraram redução do consumo de $\mathrm{O}_{2}$ no Estado III da respiração mitocondrial nas áreas isquêmicas dos animais do grupo submetido a isquemia de 60 minutos e 10 minutos de reperfusão, quando comparadas às áreas correspondentes do hemisfério não isquêmico, resultados estes menos expressivos quando comparados com o efeito da isquemia focal na respiração mitocondrial no tecido cerebral de ratos ${ }^{12,16}$.

Comparando-se os dois estudos realizados no gato, nossos resultados foram um pouco mais expressivos. Explicações para este fato podem ser a ampla variação anatômica na origem da artéria cerebral média no gato 
que poderia ter causado dificuldade na padronização da área de isquêmica obtida e possível variações na intensidade da isquemia na área selecionada para colheita da amostra, possibilidade pouco provável porque em ambos os estudos a oclusão do vaso foi efetuada de maneira semelhante. No presente trabalho o anestésico empregado foi o thionembutal, que também pode ter tido um efeito neuroprotetor. Além disso, como o controle da temperatura corporal do animal não foi completamente confiável em todos os animais e como anestesia geral pode causar hipotermia ${ }^{6}$, quedas significativas da temperatura podem ter exercido um efeito neuroprotetor, contribuindo para os efeitos pouco expressivos da isquemia sobre a função mitocondrial. Outra hipótese mais interessante é o fato de que a duração máxima da isquemia (60 minutos) pode não ter sido suficiente para provocar danos importantes nas mitocôndrias destes animais.

A reperfusão de um tecido isquêmico restaura o aporte de oxigênio e de glicose às células e restabelece a fosforilação oxidativa, o que contribui para a normalização dos processos energéticos fisiológicos celulares; porém quando a cadeia de transporte de elétrons mitocondriais mantem-se comprometida, a reperfusão tecidual desencadeia reações bioquímicas que aceleram o processo de glicólise, aumentando a acidose láctica e desencadeiam a produção de radicais livres tóxicos, culminando com a inibição da respiração mitocondrial ${ }^{17}$. Este processo é denominado de "lesão de reperfusão" ${ }^{\prime \prime 18,19}$.

Os estudos em modelos experimentais de isquemia global completa ou incompleta seguido de reperfusão em ratos, mostraram que a reperfusão de curta duração $\left(<30^{\prime}\right)$ propicia a recuperação total ou parcial do consumo de $\mathrm{O}_{2}{ }^{17,19}$, e que a reperfusão prolongada reduz novamente o consumo de $\mathrm{O}_{2}{ }^{17,20-23}$. Não encontramos na literatura estudos experimentais com isquemia cerebral focal seguida de reperfusão de curta duração. Os estudos com reperfusão de longa duração $(+$ de 1 hora), são conflitantes, pois alguns autores observam recuperação total do consumo de $\mathrm{O}_{2}$ no estado $\mathrm{III}^{24}$, e outros referem persistência de redução do consumo de $\mathrm{O}_{2}^{24}$.

Isquemias transitórias com períodos de reperfusão em ratos parecem inicialmente menos danosos ao tecido cerebral quando comparadas à isquemia continua com tempo semelhante a soma dos períodos de isquemia intermitente, ${ }^{16,26}$ porém tardiamente a performance dos animais foi semelhante ${ }^{16}$.

De uma maneira geral, a reperfusão parece não interferir com a função mitocondrial em tecidos cerebrais submetidos até 30 minutos de isquemia, porém períodos mais longos de isquemia fazem com que a retomada da função mitocondrial não seja completa com os níveis de consumo de $\mathrm{O}_{2}$, mantendo-se abaixo do período pré-isquêmico $0^{6,17,20,21,25}$.

Em nosso estudo a reperfusão foi efetuada durante 10 minutos, após os períodos variáveis de isquemia. As alterações ocorreram apenas no Estado III da função mitocondrial do grupo de animais submetidos ao tempo mais longo de isquemia (60 minutos). Parece-nos portanto com base em nossos resultados e nas observações da literatura que a duração da isquemia foi efetivamente determinante das lesões mitocondriais observadas e que a reperfusão por ser de curta duração não deve ter exercido um papel determinante.

\section{CONCLUSÃO}

Com base nos resultados obtidos pode-se concluir que o tempo de duração da isquemia foi um fator determinante na alteração da respiração mitocondrial de gatos submetidos à isquemia e reperfusão com alterações significativas aparecendo apenas nos animais submetidos a 60 minutos de isquemia seguidos de 10 minutos de reperfusão.

\section{REFERÊNCIAS}

01. White BC, Sullivan JM, Degracia DJ, O' Neill BJ, Neumar RW, Grossman LI, Rafols JA, Krause GS. Brain ischemia and reperfusion: molecular mechanisms of neuronal injury. J Neurol Sci 2000;79:1-33.

02. Ginsberg MD, Busto RBS. Rodent models of cerebral ischemia. Stroke 1989;20: 1627-42

03. The Canadian Cooperative Study Group. A randomized trial of aspirin and sulfinpyrazone in threatened stroke. N Engl J Med 1978;299:53-9.

04. Lai JCK, Chark, J.B. Preparation of synaptic and nonsynaptic mitochondria from mammalian brain. Methods Enzimol 1974;60:51-64.

05. Curti C. Uyemura SA. Estudos da fosforilação oxidativa. In: Castro e Silva O Jr., Zucoloto S, Beer A. Jr.. Modelos Experimentais de Pesquisa em Cirurgia. São Paulo: Robe Editorial. 1998. p.149-58.

06. Carlotti Jr. CG. Estudo da respiração mitocondrial do córtex de ratos submetidos à isquemia focal pela oclusão da artéria cerebral média. [Tese de Doutorado]. Faculdade de Medicina de Ribeirão Preto, Universidade de São Paulo; Ribeirão Preto; 1998.

07. Siesjö BK, Siesjö P. Mechanisms of secondary brain injury. E J Anaesthesiol 1996;13:247-68.

08. Granato RG, Ceneviva R, Souza MEJ, Picinato, MANC, Franco CFF, Castro e Silva, O Jr. Coração ictérico: avaliação da capacidade energética. Acta Cir Bras 1996;11:30-1, 1996.

09. Kreisel U. Avaliação comparativa de mitocôndrias de miocárdio de coelhos submetidos à cardioplegia básica. [Tese de Doutorado]. Faculdade de Medicina de Ribeirão Preto, Universidade de São Paulo; Ribeirão Preto; 1996.

10. Tucci S Jr., Roselino JES, Nakazato NH, Velludo MAS, Franco CFF, Souza EJ, Picinato MANC. Alterações da função mitocondrial em ratos tratados com ciclosporina. J Bras Nefrol $1993 ; 26: 657-8$. 
11. Castro e Silva O Jr.. Função mitocondrial do fígado durante a obstrução biliar extra-hepática e após a drenagem cirúrgica das vias biliares. Efeito da clorpromazina. [Tese de Doutorado]. Faculdade de Medicina de Ribeirão Preto, Universidade de São Paulo; Ribeirão Preto; 1986.

12. Carlotti CG Jr., Colli BO, KAZUO JY. Avaliação da isquemia cerebral pela respiração mitocondrial - modelo experimental. Arq Neuropsiquiat 2001;59:335-71.

13. Dias LAA, Colli BO, Coutinho Neto J, Lachat JJ. Avaliação da isquemia cerebral focal induzida pela oclusão da artéria cerebral média e a ação neuroprotetora do cetoprofeno em ratos. Arq Neuropsiquiatr 2000;5:1047-54.

14. Brandão Câmara RL. Avaliação comparativa do intumescimento osmótico (Sweeling) mitocondrial entre amostras cerebrais isquêmicas e não isquêmicas e em relação a outros tecidos (músculo cardíaco e esqulético, fígado e rim) em gatos submetidos à oclusão da artéria cerebral média direita. [Tese de Doutorado]. Faculdade de Medicina de Ribeirão Preto, Universidade de São Paulo; Ribeirão Preto; 2002.

15. Church J, Zeman S, Lodge D. The neuroprotective action of ketamine and MK-801 after transient cerebral ischemia in rats. Anesthesiology 1988;69:702-9.

16. Kurokawa Y, Tranmer BI. Interrupted arterial occlusion reduces ischemic damage in a focal cerebral ischemia model of rats. Neurosurgery 1995;37:750-7.

17. Fiskum G. Mitocondrial damage during cerebral ischemia. Ann Emerg Med 1985;14:810-5.

18. Hallenbeck JM, Dutra AJ. Backoground review and current concepts of reperfusion injury. Arch Neurol 1990;47:1245-54.

19. Aronowski J, Strong R, Grotta JC. Reperfusion injury demonstration of brain damage produced by reperfusion after transient focal ischemia in rats. Journal of Cereb Blood Flow Metab 1997; 17:1048-56.

20. Nakahara I, Haruhiko K, Taki W, Nishi S, Kito M, Yonekawa Y, Goto Y, Ogata N. Changes in major phospholipids of mitochondria during posischemic reperfusion in rat brain. $\mathrm{J}$ Neurosurg 1992;76:244-50.

21. Phillis JW, O'regan MH, Estevez AY, Song D, Vanderheide SJ. Cerebral energy metabolism during severe ischemia of varying duration and following reperfusion. J Neurochem 1996;67:1525-31.

22. Sims NR, Williams VK, Zaidan E, Powell, JA. The antioxidant defences of brain mitochondria during short-term forebrain ischemia and recirculation in the rat. Brain Res Mol Brain Res 1998;60:141-9.

23. Li AL, Kristian T, He QP, Siesjö BK. Cyclosporin A enhances survival ameliorates brain damage, and prevents secondary mitochondrial dysfunction after a 30-minutes period of transiet cerebral ischemia. Exp Neurol 2000;165:153-63.

24. Anderson MF, Sims NR. Mitochondrial respiratory function and celld eath in focal cerebral ischemia. J Neurochem 1999;73:1189-99.

25. Kuroda S, Katsura K, Hillered L, Bates TE, Siesjö BK. Delayed treatment with alpha-phenyl-N-tert-butyl nitrone (PBN) attenuates secondary mitochondrial dysfunction after transiet focal cerebral ischemia in the rat. Neurobiol Dis 1996;3:149-7.

26. Goldman MS, Anderson RE, Meyer FB. Effects of intermittent reperfusion during temporal focal ischemia. J Neurosurg 1992;77:911-6.

\section{Endereço para Correspondência:}

\section{Benedicto Oscar Colli}

Departamento de Cirurgia e Anatomia - HCFMRP

Campus Universitário USP

14048-900 Ribeirão Preto - SP

E-mail: bocolli@fmrp.usp.br 